\title{
Morphologic and immunohistochemical features of malignant peripheral nerve sheath tumors and cellular schwannomas
}

\author{
Melike Pekmezci ${ }^{1}$, David E Reuss ${ }^{2}$, Angela C Hirbe ${ }^{3}$, Sonika Dahiya ${ }^{4}$, David H Gutmann ${ }^{5}$, \\ Andreas von Deimling ${ }^{2}$, Andrew E Horvai ${ }^{6}$ and Arie Perry ${ }^{1,7}$ \\ ${ }^{1}$ Division of Neuropathology, Department of Pathology, University of California San Francisco, \\ San Francisco, CA, USA; ${ }^{2}$ Department Neuropathology, German Cancer Consortium (DKTK), CCU \\ Neuropathology German Cancer Research Center (DKFZ), Institute of Pathology, University of Heidelberg, \\ Heidelberg, Germany; ${ }^{3}$ Division of Medical Oncology, Department of Medicine, Washington University \\ School of Medicine, St Louis, MO, USA; ${ }^{4}$ Department of Pathology and Immunology, Washington University \\ School of Medicine, St Louis, MO, USA; ${ }^{5}$ Department of Neurology, Washington University School of \\ Medicine, St Louis, MO, USA; ${ }^{6}$ Department of Pathology, University of California San Francisco, \\ San Francisco, CA, USA and ${ }^{7}$ Department of Neurological Surgery, University of California San Francisco, \\ San Francisco, CA, USA
}

Cellular schwannoma is an uncommon, but well-recognized, benign peripheral nerve sheath tumor, which can be misdiagnosed as malignant peripheral nerve sheath tumor. To develop consensus diagnostic criteria for cellular schwannoma, we reviewed $\mathbf{1 1 5}$ malignant peripheral nerve sheath tumor and $\mathbf{2 6}$ cellular schwannoma cases from two institutions. Clinical data were retrieved from the electronic medical records, and morphologic features, maximal mitotic counts, Ki67 labeling indices, and immunohistochemical profiles (SOX10, SOX2, p75NTR, p16, p53, EGFR, and neurofibromin) were assessed. Several features distinguish cellular schwannoma from malignant peripheral nerve sheath tumor. First, in contrast to patients with malignant peripheral nerve sheath tumor, no metastases or disease-specific deaths were found in patients with cellular schwannoma. More specifically, 5 -year progression-free survival rates were 100 and $18 \%$, and 5 -year disease-specific survival rates were 100 and $32 \%$ for cellular schwannoma and malignant peripheral nerve sheath tumor, respectively. Second, the presence of Schwannian whorls, a peritumoral capsule, subcapsular lymphocytes, macrophage-rich infiltrates, and the absence of fascicles favored the diagnosis of cellular schwannoma, while the presence of perivascular hypercellularity, tumor herniation into vascular lumens, and necrosis favor malignant peripheral nerve sheath tumor. Third, complete loss of SOX10, neurofibromin or p16 expression, or the presence of EGFR immunoreactivity was specific for malignant peripheral nerve sheath tumor $(\boldsymbol{P}<0.001$ for each). Expression of p75NTR was observed in $80 \%$ of malignant peripheral nerve sheath tumors compared with $31 \%$ of cellular schwannomas $(P<0.001)$. Fourth, Ki-67 labeling indices $\geq \mathbf{2 0 \%}$ were highly predictive of malignant peripheral nerve sheath tumor $(87 \%$ sensitivity and $96 \%$ specificity). Taken together, the combinations of these histopathological and immunohistochemical features provide useful criteria to distinguish between malignant peripheral nerve sheath tumor and cellular schwannoma with high sensitivity and specificity. Additional retrospective and prospective multicenter studies with larger data sets will be required to validate these findings.

Modern Pathology (2015) 28, 187-200; doi:10.1038/modpathol.2014.109; published online 5 September 2014

Correspondence: Dr M Pekmezci, MD, Division of Neuropathology, Department of Pathology, University of California, San Francisco (UCSF), 505 Parnassus Avenue, \#M551, Box\# 0102, San Francisco, CA 94143, USA.

E-mail: melike.pekmezci@ucsfmedctr.org

Received 24 April 2014; revised 23 June 2014; accepted 23 June 2014; published online 5 September 2014
Malignant peripheral nerve sheath tumors are malignant neoplasms arising from peripheral nerve or extraneural soft tissue that display evidence of nerve sheath differentiation. ${ }^{1}$ However, due to the lack of sensitive and specific diagnostic markers, the differential diagnosis is often extensive, including 
various sarcomas and benign peripheral nerve sheath tumors, each with different clinical outcomes and therapeutic options. For individuals harboring a malignant peripheral nerve sheath tumor, despite adjuvant chemotherapy and radiation, the overall prognosis is poor, with 5-year disease-specific survival ranging between 35 and $50 \% .^{2-4}$ In a large cohort, $41 \%$ of patients who initially presented with a localized malignant peripheral nerve sheath tumor developed distant metastasis in a median time of 12 months. ${ }^{2}$

Cellular schwannoma initially described by Woodruff et $a l^{5}$ is an uncommon, but wellrecognized, benign nerve sheath tumor that often presents diagnostic challenges. Cellular schwannoma accounts for $2.8-5.2 \%$ of all peripheral nerve sheath tumors, ${ }^{6-8}$ and worrisome features commonly include increased cellularity, frequent mitoses, and locally destructive growth. ${ }^{9}$ Despite these alarming features, cellular schwannoma lacks metastatic potential and local recurrence is variable $(8-23.4 \%))^{8,10}$ Nevertheless, an erroneous diagnosis of malignancy, most commonly malignant peripheral nerve sheath tumor, is established in $16-28 \%$ of reported cases. ${ }^{8,10}$

Given dramatically different prognostic and therapeutic implications, distinguishing cellular schwannoma from malignant peripheral nerve sheath tumor is critical. The presence of foamy macrophages, a well-formed capsule containing lymphoid aggregates, diffuse strong S100 protein staining and pericellular collagen IV expression support a diagnosis of cellular schwannoma. ${ }^{11}$ Other potentially useful markers, such as SOX10 (SRY-related HMG box 10) and p16, have only been studied in the setting of malignant peripheral nerve sheath tumor versus other sarcomas, ${ }^{12-14}$ whereas putative neural stem cell markers (SOX2 and p75 neurotrophin receptor (p75NTR)) have not been systematically explored. ${ }^{15}$ Similarly, the neurofibromatosis type 1 protein product (neurofibromin), p53 and EGFR expression, implicated in malignant peripheral nerve sheath tumor pathogenesis, have only rarely been examined in cellular schwannoma. ${ }^{2,16}$ In the current study, we evaluated the utility of SOX10, SOX2, p75NTR, p16, p53, EGFR, and neurofibromin expression by immunohistochemistry in the differential diagnosis of malignant peripheral nerve sheath tumor versus cellular schwannoma, additionally assessing their prognostic roles in the setting of malignancy.

\section{Materials and methods}

\section{Case Selection}

This study was performed under approved Human Studies Protocols at the University of California, San Francisco (The Committee on Human Research; UCSF) and Washington University in St Louis (Institutional Review Board; WUSTL). UCSF cases were identified using a pathology database search for the diagnoses of malignant peripheral nerve sheath tumor and cellular schwannoma between June 1990 and June 2012. All cases with diagnoses of 'malignant peripheral nerve sheath tumor,' 'malignant schwannoma,' 'malignant neurilemmoma,' 'neurofibrosarcoma,' 'nerve sheath sarcoma,' 'cellular schwannoma,' and 'cellular neurilemmoma' were reviewed. In addition, all cases of 'schwannoma,' and 'neurilemmoma' with mention of 'cellular' in the comment or discussion sections were reviewed to identify additional cellular schwannoma cases. Clinical data were retrieved from electronic medical records. Three pathologists (MP, AEH, and AP) independently reviewed all cases with available material for diagnostic confirmation, and achieved a consensus diagnosis. Schwannomas composed almost exclusively of Antoni A areas with increased cellularity were diagnosed as cellular schwannoma, and those with $>10 \%$ Antoni B areas and well-defined Verocay bodies were excluded. Outside consultation cases, cutaneous tumors in which melanoma was a consideration, neoplasms in which other sarcoma diagnoses (eg, leiomyosarcoma and fibromyxoid sarcoma) remained in the differential diagnosis, and nerve sheath tumors with equivocal features of malignancy were excluded. The search thus yielded a cohort of 40 malignant peripheral nerve sheath tumor and 12 cellular schwannoma from UCSF. All study cases were negative for SS18 gene rearrangement by fluorescence in situ hybridization.

Cellular schwannoma cases from WUSTL were identified using a pathology database search, and three pathologists (MP, SD, and AP) independently reviewed the cases with available material using the same criteria as above. A consensus diagnosis was achieved in 14 cellular schawannoma cases, which were included in the study. In addition, a tissue microarray block containing 75 malignant peripheral nerve sheath tumor cases (previously confirmed by AP) was included in the study. The tissue micro array was created using $0.6 \mathrm{~mm}$ cores in duplicate or triplicate from areas of greatest interest from each case, and was previously validated. ${ }^{17}$

\section{Histological Features and Grading}

Cases with whole slide sections were reviewed for histologic features including the presence of a peritumoral capsule, whorls, fascicles, inflammatory cells, perivascular hypercellularity, herniation of tumor into vessels, necrosis, and mitotic activity. For the quantification of mitotic activity, once a mitotic figure is identified, we counted mitotic figures in 10 consecutive high-power fields $\left(0.24 \mathrm{~mm}^{2}\right)$. This was repeated in up to 10 different regions as tumor size permits, and the highest number was recorded. Malignant peripheral nerve sheath tumors were stratified into low and high 
grades based on the previously published criteria ${ }^{18}$ with 'high grade' corresponding to tumors with hypercellularity, cytologic anaplasia, and a maximal mitotic index greater than 6/10 high-power fields, often accompanied by necrosis. Previously performed grading was used for malignant peripheral nerve sheath tumors from WUSTL, and mitotic activity was not calculated.

\section{Immunohistochemistry}

A representative block was chosen for immunohistochemistry from all UCSF cases and WUSTL cellular schwannoma cases, and 5-micron paraffin sections were stained using standard techniques (Table 1). Whole section slides were not available for malignant peripheral nerve sheath tumor cases from WUSTL; therefore 5-micron sections from the tissue microarray were used for immunohistochemistry. Due to limited amount of tissue only selected stains could be performed on these cases. Expression in $90 \%$ or more of the tumor cells was considered as diffuse positivity, while expression in $<5 \%$ was considered as negative for all stains except Ki-67. Each tissue microarray core was independently evaluated and assigned a percent-staining value. Cases with significant variation from one to core the other were re-evaluated, and average staining percentage was calculated. The number of cases that would potentially be misclassified (as negative/ positive or patchy/diffuse) if we had a single core due to heterogeneity in staining was limited to two cases for S100, three cases for SOX10, two cases for p16, and one case for EGFR. Ki-67 labeling index was estimated as percent positive nuclei in the area of highest labeling using online software (ImmunoRatio ${ }^{(}$, version 1.0c, Tampare, Finland), Ki-67 stain was not performed for malignant peripheral nerve sheath tumors from WUSTL.

\section{Statistical Analyses}

Statistical analyses were performed using the SPSS 22.0.0 software package (SPSS, Chicago, IL, USA).
Descriptive data were presented as medians with ranges. Comparisons among categorical variables were performed with the Fisher's exact test, and comparisons of continuous variables were performed with Mann-Whitney $U$ test. Survival analyses were performed using Kaplan-Meier analysis, with the log-rank (Mantel-Cox) method to compare survival curves. Receiver operating characteristics curves were created to assess the performance of each stain. Bonferroni correction was applied for seven stains evaluated in this study, and a $P$-value of $<0.0071$ was set as significant.

\section{Results}

In total, 115 malignant peripheral nerve sheath tumor cases (50 males, 44\%) and 26 cellular schwannoma cases (12 males, $46 \% ; P=0.83$ ) were included in this analysis. Median age at initial diagnosis was 38.2 years (range: 0.1-81.6) for malignant peripheral nerve sheath tumor and 44.9 years (range: 13.8-70.3) for cellular schwannoma (overall 39.4; range: 0.1-81.6; $P=0.212$ ).

Fifty-one malignant peripheral nerve sheath tumor patients had a clinical diagnosis of neurofibromatosis 1 based on the established diagnostic criteria. ${ }^{19}$ After review of all available clinical and pathology reports, 24 malignant peripheral nerve sheath patients were classified as sporadic, while the remaining 40 cases were excluded from neurofibromatosis status-related analyses due to lack of sufficient clinical information for accurate classification. One cellular schwannoma patient was diagnosed with neurofibromatosis 2, while another was diagnosed with familial schwannomatosis. There was an additional patient with cellular schwannoma, who was reported to have some form of neurofibromatosis; however, it was unclear from the available clinical notes whether this represented neurofibromatosis 1 or 2. Median ages at diagnosis were 33.8 years (range: 11.2-77.5) and 41.7 years (range: 9.4-81.4) for malignant peripheral nerve sheath tumor patients with and without clinical diagnosis of neurofibromatosis 1 ,

Table 1 Conditions for immunohistochemical stains

\begin{tabular}{|c|c|c|c|c|c|}
\hline Marker & Clone & & Source & Dilution & Antigen retrieval \\
\hline S100 & S100 & Rabbit polyclonal & Ventana & $1: 2$ & CC1-120 min \\
\hline SOX10 & SOX10 & Rabbit polyclonal & Cell Marque & $1: 50$ & CC1-60 min \\
\hline Neurofibromin & NFC & Mouse monoclonal & DKFZ & $1: 4$ & CC2-56 min \\
\hline SOX2 & AB5603 & Rabbit polyclonal & EMD Millipore & $1: 200$ & CC1-32 min \\
\hline p75NTR & NGFR5 & Mouse monoclonal & Abcam & $1: 100$ & CC1-32 min \\
\hline p16 & JC8 & Mouse monoclonal & Santa Cruz & $1: 50$ & CC1-32 min \\
\hline p53 & DO-7 & Mouse monoclonal & Dako & $1: 50$ & CC1-32 min \\
\hline EGFR & 3C6 & Mouse monoclonal & Ventana & $1: 2$ & CC1-32 min \\
\hline MIB1 & $30-9$ & Rabbit monoclonal & Ventana & $1: 1$ & CC1-32 min \\
\hline
\end{tabular}

Abbreviations: CC1, Cell conditioner 1 (pH 9), Ventana (Ventana Medical Systems, Tucson, AZ, USA); CC2, Cell conditioner 2 (pH 6), Ventana (Ventana Medical Systems); min, minutes. 
respectively $(P=0.04)$. In general, patients with malignant peripheral nerve sheath tumor had larger tumors $(8.8 \mathrm{~cm}$, range: $0.5-26)$ than patients with cellular schwannoma $(4.8 \mathrm{~cm}$, range: $1-9.8$; $P=0.005$ ), although the ranges overlapped considerably. In all, 3 cellular schwannomas $(12 \%)$ and 34 malignant peripheral nerve sheath tumors $(31 \%)$ were located in the extremities, while the remaining were found in paraspinal sites, brachial/sacral plexi, and intracranial locations $(P=0.053)$. Median follow-up was 15.4 (range: 1.1-180) months for malignant peripheral nerve sheath tumor and 16.4 (range: 0.1-202.5) months for cellular schwannoma $(P=0.495)$. There was no significant difference between tumors resected at the two institutions with respect to clinical features ( $P>0.05$ for all; Table 2$)$.

Among malignant peripheral nerve sheath tumors, $13(11 \%)$ were low grade and 102 (89\%) high grade, including $20 \%$ diagnosed as malignant Triton tumors. The extent of resection was extracted from operative reports and imaging, demonstrating that 26 malignant peripheral nerve sheath tumor patients $(65 \%)$ underwent gross-total resection. The microscopic surgical margin status was available in 36 cases, of which 13 were negative (36\%). Rates for adjuvant radiation, chemotherapy, and combined chemo-radiation were 28,15 , and $13 \%$, respectively. There were $10(17 \%)$ patients who had metastasis at the time of initial diagnosis, and 12 additional patients who developed metastases during followup (median 16.8 months, range: 5.3-57.1). The most common site of metastasis was lung (12 patients, $55 \%)$. Thirty-six patients suffered from recurrent/ progressive disease with a median time of 10.3 months (range: 1.4-105). Sixty-five patients died as a result of their malignant peripheral nerve sheath tumor, one patient died of high-grade lymphoma, and 49 patients were alive at the last visit (median 32.6 months, range 1.1-180 months). Median time to death for those who died of disease was 16 months (range: 0.4-129.3).
Among the patients with diagnosis of cellular schwannoma, seven $(27 \%)$ were originally diagnosed as low-grade malignant peripheral nerve sheath tumor. Ten $(83 \%)$ patients underwent grosstotal resection and surgical margins were microscopically negative in four of the nine patients $(44 \%)$ with available data. Twenty-five percent of the patients received adjuvant radiation treatment. None of the patients had metastasis during the follow-up period, and one patient (4\%) had a recurrence 10 years after initial resection. Twentyfive patients $(96 \%)$ were alive with no evidence of disease at their last visit (median 16.9 months, range 0.1-202.5), while one patient died of metastatic pancreatic adenocarcinoma 16 months later. Overall, cellular schwannoma patients had significantly better progression-free and disease-specific survival times than malignant peripheral nerve sheath tumor patients $(P<0.001$, for both; Figure 1a and b). Fiveyear progression-free survival rates were $17.7 \%$ for malignant peripheral nerve sheath tumor and $100 \%$ for cellular schwannoma, and 5-year disease-specific survival rates were $31.9 \%$ for malignant peripheral nerve sheath tumor and $100 \%$ for cellular schwannoma. There were no differences between the two institutions regarding progression-free survival and disease-specific survival ( $P>0.05$, for both).

Common morphologic features for malignant peripheral nerve sheath tumor and cellular schwannoma are illustrated in Figure 2 and summarized in Table 3. In brief, the presence of Schwannian whorls, a peritumoral capsule, subcapsular lymphocytes, macrophage-rich infiltrates, and an absence of fascicles favor the diagnosis of cellular schwannoma $(P<0.005$ for all). In contrast, the presence of perivascular accentuation of cellularity, tumor herniation into vascular spaces, and necrosis favor malignant peripheral nerve sheath tumor $(P<0.004$ for all). The median values for maximal mitotic counts per 10 consecutive high-power fields were 25 (range: 1-90) and 2.5 (range: 1-10) for malignant

Table 2 Clinical features of UCSF and WUSTL case cohorts

\begin{tabular}{|c|c|c|c|c|c|c|c|}
\hline & \multicolumn{3}{|c|}{ UCSF cohort } & \multicolumn{4}{|c|}{ WUSTL cohort } \\
\hline & $\begin{array}{l}\text { Malignant peripheral } \\
\text { nerve sheath tumor }\end{array}$ & $\begin{array}{c}\text { Cellular } \\
\text { schwannoma }\end{array}$ & $\mathrm{P}^{\mathrm{a}}$ & $\begin{array}{l}\text { Malignant peripheral } \\
\text { nerve sheath tumor }\end{array}$ & $\begin{array}{c}\text { Cellular } \\
\text { schwannoma }\end{array}$ & $\mathrm{P}^{\mathrm{a}}$ & $\mathrm{P}^{\mathrm{b}}$ \\
\hline Gender; male (\%) & $17(43 \%)$ & $6(50 \%)$ & 0.75 & $33(44 \%)$ & $6(43 \%)$ & 0.83 & 0.55 \\
\hline Age, years ${ }^{\mathrm{c}}$ & $37.5(11.2-71.8)$ & $44.7(13.8-70.3)$ & 0.14 & $38.3(0.1-81.6)$ & $46(16-70)$ & 0.6 & 0.52 \\
\hline$<40$ years old $(\%)$ & $23(58 \%)$ & $5(42 \%)$ & 0.51 & $39(52 \%)$ & $6(43 \%)$ & 0.57 & 0.73 \\
\hline NF $1(\%)$ & $25(64 \%)$ & 0 & $<0.0001$ & $22(82 \%)$ & $1^{\mathrm{d}}(7 \%)$ & $<0.0001$ & 0.074 \\
\hline NF $2(\%)$ & 0 & $1(8 \%)$ & & 0 & $1^{d}(7 \%)$ & & \\
\hline Size $(\mathrm{cm})^{\mathrm{C}}$ & $8.8(0.5-26)$ & $4.8(1-9.8)$ & 0.005 & & & & \\
\hline Site; extremity (\%) & $12(30 \%)$ & 0 & 0.047 & $22(31 \%)$ & $3(21 \%)$ & 0.75 & 0.55 \\
\hline
\end{tabular}

Abbreviations: NF, neurofibromatosis; UCSF, University of California San Francisco; WUSTL, Washington University in St Louis.

$P$-values in bold are statistically significant.

${ }^{\text {a }} P$-value belongs to comparisons between MPNSTs and CSs.

${ }^{\mathrm{b}} P$-value belongs to comparisons between two institutions.

${ }^{\mathrm{C}}$ Values are median (range).

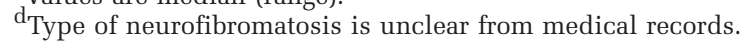


a

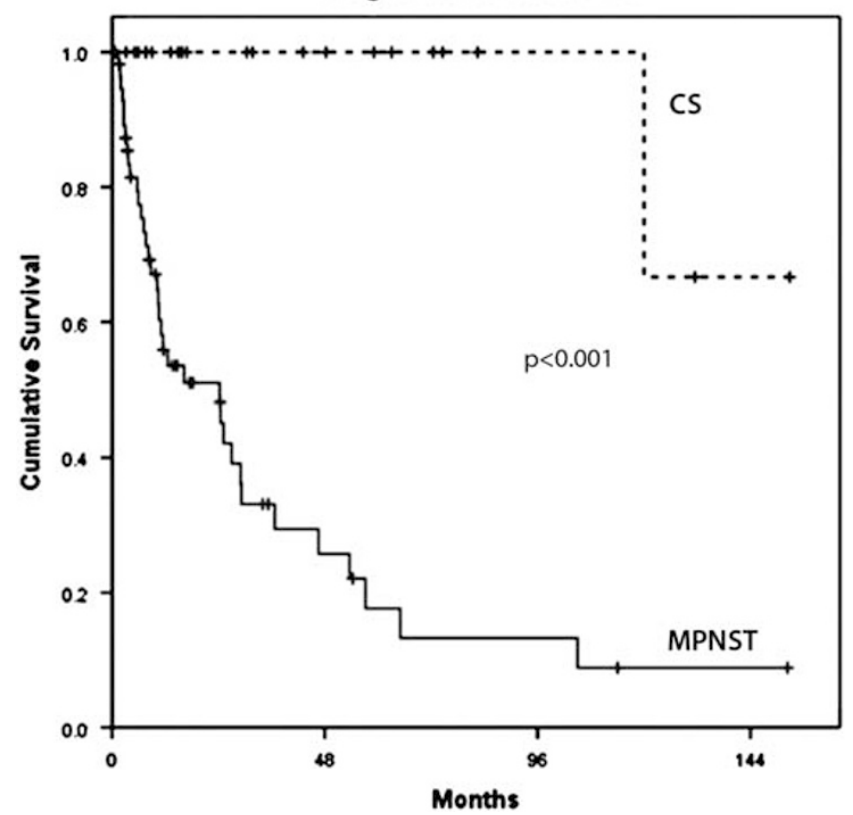

b

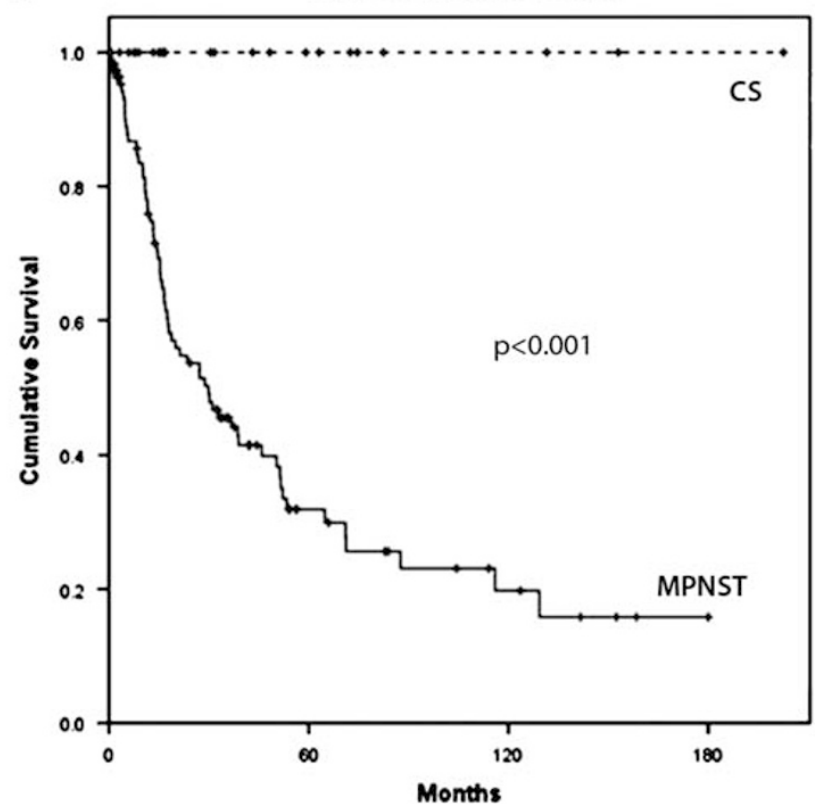

Figure 1 Survival characteristics of malignant peripheral nerve sheath tumors and cellular schwannomas. Kaplan-Meier curves demonstrate significant difference between malignant peripheral nerve sheath tumors and cellular schwannomas for progression-free survival (a) and disease-specific survival (b).

peripheral nerve sheath tumor and cellular schwannoma, respectively $(P<0.0001)$. A cutoff value of $\geq 10$ mitoses per 10 high-power fields yielded $82.5 \%$ sensitivity and $96.1 \%$ specificity for malignant peripheral nerve sheath tumor (area under curve $=0.920 \quad(95 \%$ confidence interval: $0.853-$ 0.986)). There was only one cellular schwannoma with 10 mitoses per 10 high-power fields and that patient was free of disease at the end of the study (59 months follow-up).

Immunohistochemical features of malignant peripheral nerve sheath tumor and cellular schwannoma are listed in detail (Table 3), and selected stains illustrated in Figures 3 and 4 . In brief, S100 and SOX10 expression was focally positive in all cellular schwannomas, but in only a fraction of malignant peripheral nerve sheath tumors (41 and $18 \%$, respectively, $P<0.001)$. When positive, S100 immunoreactivity was usually patchy in malignant peripheral nerve sheath tumors and was usually diffuse in cellular schwannomas. In addition, diffuse SOX10 expression was limited to cellular schwannoma cases. All cellular schwannomas retained neurofibromin expression, whereas 25 of 37 (68\%) malignant peripheral nerve sheath tumors tested showed complete loss of expression in tumor cells $(P<0.001)$. Intratumoral macrophages served as an internal positive control for neurofibrominnegative tumors. In addition, there were five cases of neurofibromatosis 1-associated malignant peripheral nerve sheath tumor with patchy expression (Figure 5). In one of these cases, while the highgrade component was entirely negative, the low- grade component retained diffuse expression. One case composed entirely of high-grade tumor and showed distinct areas of complete loss and scattered foci of retained expression. Three other cases, one low grade and two high grade, showed an expression pattern of highly intermingled positive and negative cells. A similar intermingled expression pattern was also identified in a cellular schwannoma. In all, 7 sporadic malignant peripheral nerve sheath tumor $(18.9 \%)$ and 24 cellular schwannoma $(96 \%)$ retained diffuse neurofibromin expression $(P<0.001)$.

The p16 immunostain was positive in all cellular schwannoma cases, and the majority of these cases contained patchy expression, while p16 was completely lost in $82(73.2 \%)$ malignant peripheral nerve sheath tumor cases $(P<0.001)$. EGFR expression was present in $31 \%$ of malignant peripheral nerve sheath tumors, while all cellular schwannomas were immunonegative $(P<0.001)$. Expression of p75NTR was present in $80 \%$ of malignant peripheral nerve sheath tumors and $30.8 \%$ of cellular schwannomas $(P<0.001)$. The median Ki-67 proliferation index was $40.3 \%$ (range: $1-84$ ) and $7.6 \%$ (range: 2-36) for malignant peripheral nerve sheath tumor and cellular schwannoma, respectively $(P<0.001)$. A cutoff value of $\geq 20 \%$ for Ki67 labeling index for the diagnosis of malignant peripheral nerve sheath tumor yielded $87.2 \%$ sensitivity and $96.2 \%$ specificity (area under curve $=0.914(95 \%$ confidence interval: 0.840-0.988)). Additionally, Ki67 labeling was often heterogeneous with 'hot spots' in cellular schwannoma, while a high labeling 

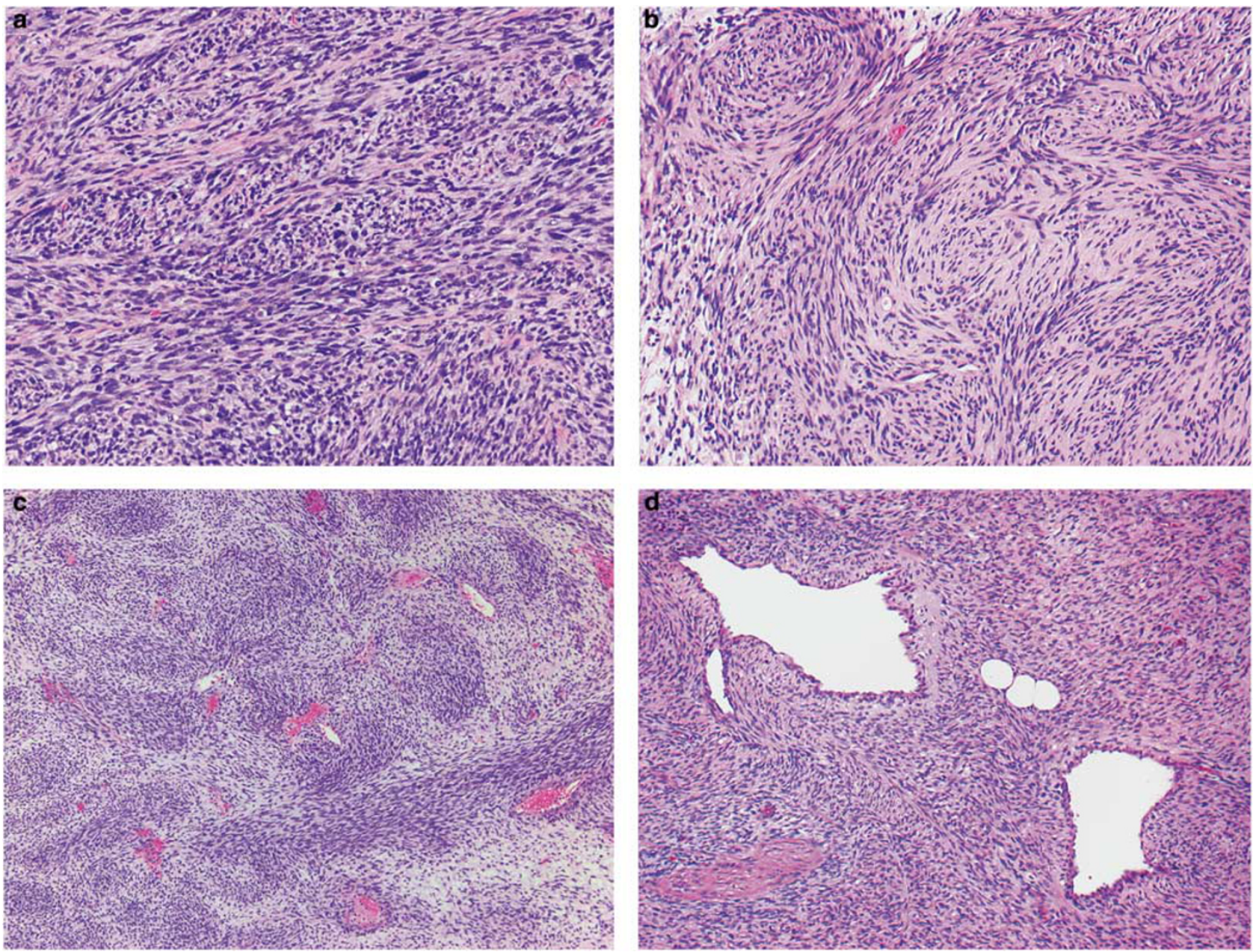

Figure 2 Selected histologic features evaluated in the study. (a) Malignant peripheral nerve sheath tumor with intersecting fascicles; (b) cellular schwannoma demonstrating numerous whorls. These whorls are strongly S100 positive (not shown) and named as 'Schwannian'; (c) malignant peripheral nerve sheath tumor exhibiting perivascular hypercellularity; (d) malignant peripheral nerve sheath tumor shows nodules of tumor cells herniating into vascular lumens.

index was typically diffuse in malignant peripheral nerve sheath tumors. The sensitivities and specificities of these immunohistochemical stains for the diagnosis of malignant peripheral nerve sheath tumor are summarized in Table 4.

Complete loss of neurofibromin expression was more common in neurofibromatosis 1-associated tumors $(79 \%)$ relative to sporadic malignant peripheral nerve sheath tumors (46\%); however, this did not reach statistical significance $(P=0.067)$. On the other hand, diffuse expression with neurofibromin was only seen in seven $(53.9 \%)$ sporadic malignant peripheral nerve sheath tumors, and none of the neurofibromatosis 1-associated malignant peripheral nerve sheath tumors $(P=0.001)$. S100 immunoreactivity was seen in $58 \%$ of sporadic and $44 \%$ of neurofibromatosis 1-associated tumors $(P=0.18)$. On the other hand, diffuse expression was seen in $22 \%$ of neurofibromatosis 1-associated tumors, and none of the sporadic tumors $(P=0.009)$. The results of the remaining immunohistochemical stains did not differ significantly between sporadic and neurofibromatosis 1-associated malignant peripheral nerve sheath tumor $(P>0.05$ for all).

Malignant peripheral nerve sheath tumors diagnosed by whole section (UCSF cases) were more likely to be positive for EGFR as compared with those diagnosed by tissue microarray (WUSTL cases) (73 and 8\%, respectively; $P<0.001$ ). Immunohistochemical frequencies of S100 and SOX10 positivity were slightly more common among malignant peripheral nerve sheath tumors diagnosed on whole section (53 and $25 \%$, respectively) as compared with tissue microarray (35 and $15 \%$, respectively); however, this did not reach statistical significance $(P=0.072$ and $P=0.21$, respectively). Frequencies of SOX2 and p16 expression were slightly more common among malignant peripheral nerve sheath tumors diagnosed on tissue microarray (32 and $87 \%$, respectively) as compared with whole section (18 and $78 \%$, respectively; $P=0.18$ and $P=0.29$, respectively). Due to limited number of 
Table 3 Morphologic and immunohistochemical findings

\begin{tabular}{|c|c|c|c|}
\hline & Malignant peripheral nerve sheath tumor & Cellular schwannoma & $\mathrm{P}$ \\
\hline \multicolumn{4}{|l|}{ Morphologic findings } \\
\hline Fascicles & $38(95 \%)$ & $16(62 \%)$ & 0.002 \\
\hline Schwannian Whorls & $2(5 \%)$ & $14(54 \%)$ & $<0.001$ \\
\hline Thick-hyalinized vessels & $2(5 \%)$ & $8(31 \%)$ & 0.010 \\
\hline Intravascular bulging & $20(50 \%)$ & $4(15 \%)$ & 0.004 \\
\hline Perivascular hypercellularity & $19(48 \%)$ & $2(8 \%)$ & 0.001 \\
\hline Capsule & $3(8 \%)$ & $15(62 \%)$ & $<0.001$ \\
\hline Subcapsular lymphocytes & $4(10 \%)$ & $11(42 \%)$ & 0.003 \\
\hline Macrophages & 0 & $7(27 \%)$ & 0.001 \\
\hline Necrosis & $31(78 \%)$ & $4(15 \%)$ & $<0.001$ \\
\hline Mitoses/10HPF; Median (range) & $25(1-90)$ & $2.5(1-10)$ & $<0.001$ \\
\hline$\geq 10$ Mitoses/10HPF & $33(83 \%)$ & $1(4 \%)$ & $<0.001$ \\
\hline \multicolumn{4}{|l|}{ Immunohistochemical stains } \\
\hline S100; positive & $47(41 \%)$ & $26(100 \%)$ & $<0.001$ \\
\hline Diffuse & $16(14 \%)$ & $25(96 \%)$ & $<0.001$ \\
\hline SOX10; positive & $21(18 \%)$ & $26(100 \%)$ & $<0.001$ \\
\hline Diffuse & 0 & $17(65 \%)$ & $<0.001$ \\
\hline Neurofibromin; positive & $12(32 \%)$ & $25(100 \%)$ & $<0.001$ \\
\hline Diffuse & $7(19 \%)$ & $24(96 \%)$ & $<0.001$ \\
\hline p16; positive & $30(27 \%)$ & $26(100 \%)$ & $<0.001$ \\
\hline Diffuse & $13(12 \%)$ & $3(12 \%)$ & 0.992 \\
\hline EGFR; positive & $35(31 \%)$ & 0 & $<\mathbf{0 . 0 0 1}$ \\
\hline Diffuse & $7(6 \%)$ & 0 & 0.348 \\
\hline p75NTR; positive & $32(80 \%)$ & $8(31 \%)$ & $<0.001$ \\
\hline SOX2; positive & $95(83 \%)$ & $25(96 \%)$ & 0.123 \\
\hline p53; positive & $30(75 \%)$ & $20(85 \%)$ & 0.766 \\
\hline Ki67, \%; median (range) & $40.3(1-84)$ & $7.6(2-36)$ & $<0.001$ \\
\hline$\geq 20 \%$ & $34(87 \%)$ & $1(4 \%)$ & $<0.001$ \\
\hline
\end{tabular}

Abbreviation: HPF, high-power fields.

$P$-values in bold are statistically significant.

available slides, immunohistochemical stains for neurofibromin, p53, and p75NTR were not performed on WUSTL malignant peripheral nerve sheath tumors.

\section{Clinical and Immunohistochemical Features of Malignant Peripheral Nerve Sheath Tumor and Their Association with Survival}

Among malignant peripheral nerve sheath tumor cases, progression-free survival and disease-specific survival were similar for males versus females, neurofibromatosis 1 versus non-neurofibromatosis 1 patients, extremity versus non-extremity sites, sub-total resection versus gross-total resection, and negative versus positive margin status $(P>0.05$ for all). Patients younger than 40 years of age had shorter median progression-free survival (10.6 months) compared with older patients (57.2 months); however, this did not reach statistical significance $(P=0.01)$. There was no difference between younger ( $<40$ years old) and older patients for disease-specific survival $(P=0.524)$.

Five-year disease-specific survival rates for lowgrade and high-grade tumors were 80 and $27.2 \%$, respectively $(P=0.007)$. Corresponding 5 -year progression-free survival rates were 51.4 and $11.6 \%$, respectively $(P=0.097)$. Five-year disease-specific survival rates were 31.3 and $37.5 \%$ for patients with and without recurrence, respectively $(P=0.883)$. On the other hand, patients without metastasis had better 5-year disease-specific survival (38.3\%) relative to those with metastasis $(5.1 \%, P=0.003)$. There was no difference in progression-free survival or disease-specific survival between cases with positive and negative expression for any of the proteins analyzed by immunohistochemistry $(P>0.0071$ for all). Detailed data regarding univariate survival analysis are presented in Tables 5 and 6.

\section{Discussion}

Cellular schwannoma is composed almost exclusively of compact spindle cells with variable hyperchromasia and pleomorphism, and the absence of Verocay bodies. In some, destructive growth, increased mitotic activity, and/or foci of necrosis lead to an overdiagnosis of malignancy, most commonly malignant peripheral nerve sheath tumor. .,9,20 $^{-19}$ In this regard, more than a quarter of our cellular schwannoma cases were initially misdiagnosed as malignant peripheral nerve sheath tumor, comparable to previous reports. ${ }^{8,10}$ The significance of malignant peripheral nerve sheath tumor misdiagnosis is underscored by the relative benign nature of cellular schwannoma, which have essentially no metastatic potential and tumor-associated 

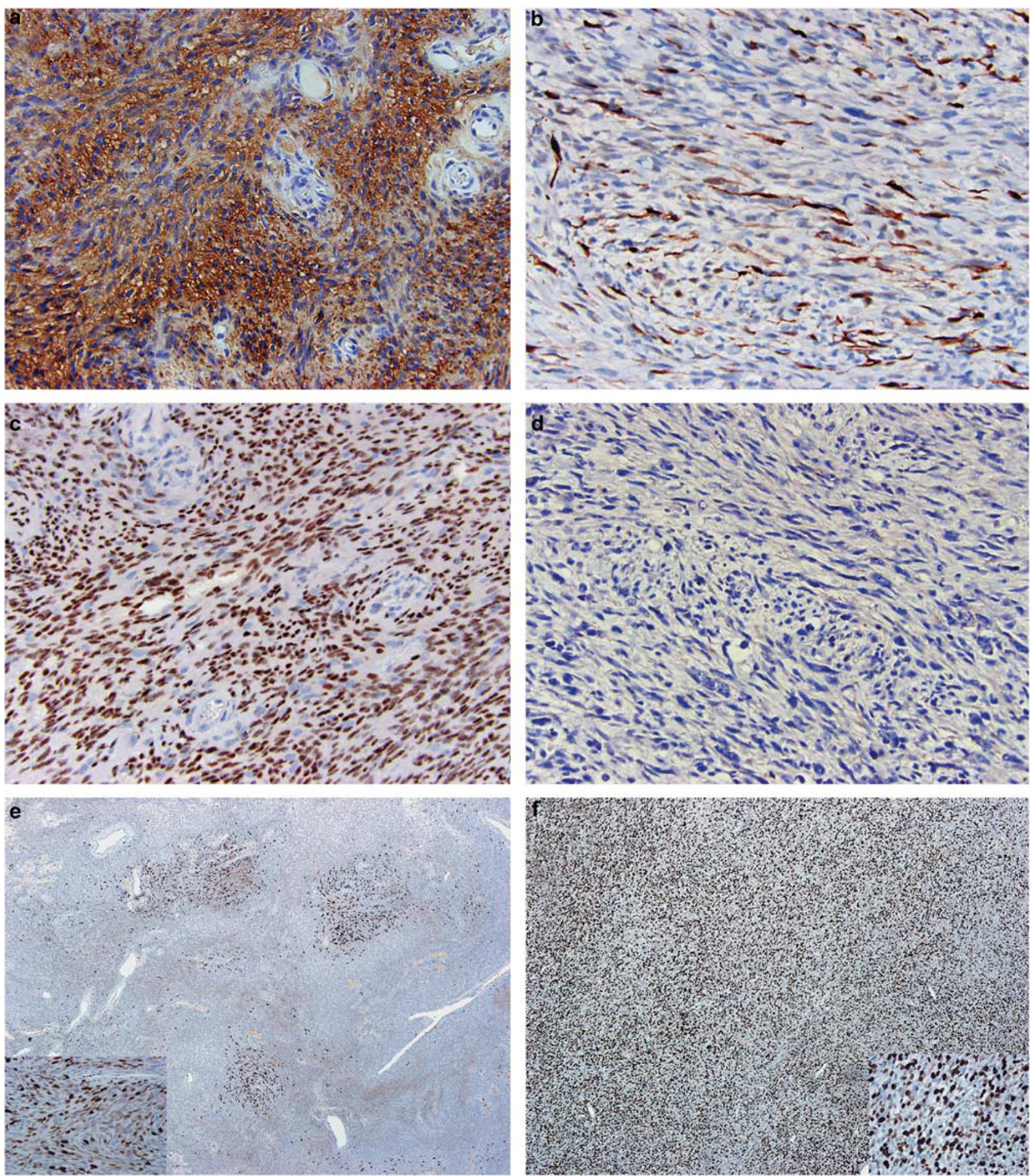

Figure 3 Immunohistochemical expression patterns of S100, SOX10, and Ki67 among malignant peripheral nerve sheath tumors and cellular schwannomas. (a) Diffuse S100 positivity in cellular schwannoma; (b) S100 highlights occasional cells within a malignant peripheral nerve sheath tumor, which are potentially entrapped Schwann cells; (c) diffuse SOX10 expression in cellular schwannoma; (d) SOX10 is negative in malignant peripheral nerve sheath tumor; (e) Ki67 stain demonstrates variable labeling indices throughout a cellular schwannoma with hot spots; inset shows high-power image of a hot spot with labeling index of $36 \%$; (f) Ki67 stain demonstrates diffusely increased labeling in a malignant peripheral nerve sheath tumor; inset shows high-power image with a labeling index of $39 \%$.

mortality. ${ }^{8,10}$ As such, we found no metastases or disease-related deaths in patients with cellular schwannoma. However, in striking contrast, $37.3 \%$ of patients with malignant peripheral nerve sheath tumors had metastases and the 5-year diseasespecific survival rate was $31.9 \%$. 

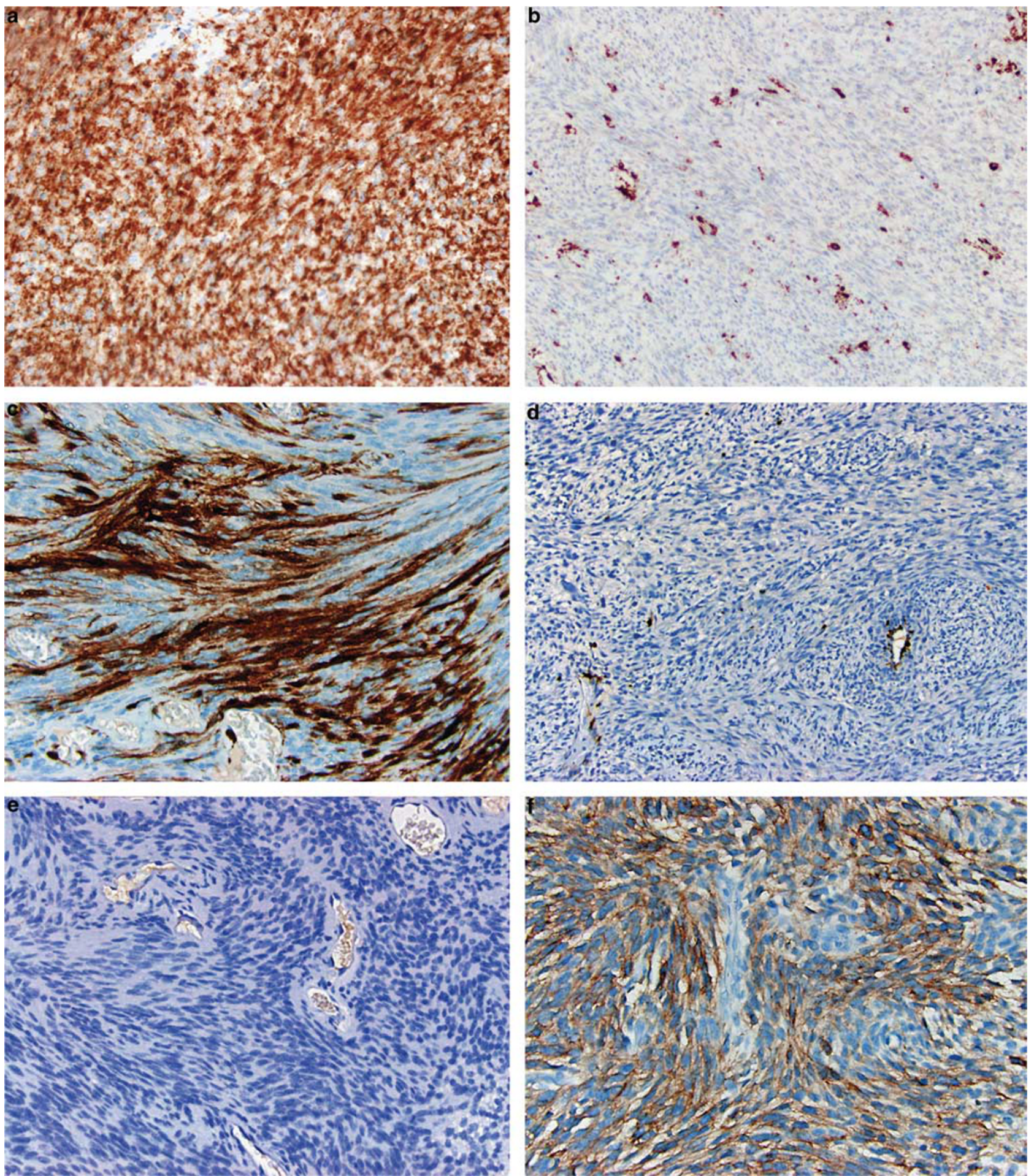

Figure 4 Immunohistochemical expression patterns of neurofibromin, p16, and EGFR among malignant peripheral nerve sheath tumors and cellular schwannomas. (a) Neurofibromin stain is retained diffusely in cellular schwannoma; (b) neurofibromin is lost in tumor cells in malignant peripheral nerve sheath tumor, and is retained in vasculature and inflammatory cells; (c) p16 stain is diffusely positive in cellular schwannoma; (d) p16 expression is lost in malignant peripheral nerve sheath tumor, and is retained in vasculature; (e) EGFR stain is negative in cellular schwannoma; (f) diffuse EGFR expression in malignant peripheral nerve sheath tumor.

Our study confirmed previously proposed morphologic features of cellular schwannoma including well-defined capsules, macrophage-rich areas, cellular 'Schwannian whorls' and hyalinized thick blood vessels. ${ }^{5,10}$ By contrast, thick intersec- ting bundles, intravascular herniation, increased perivascular cellularity, and necrosis were features of malignant peripheral nerve sheath tumors. Importantly, however, our data showed that most morphologic findings were not exclusive to either 
cellular schwannoma or malignant peripheral nerve sheath tumor. Most notably, increased mitotic activity was found in both tumor types, but mitotic

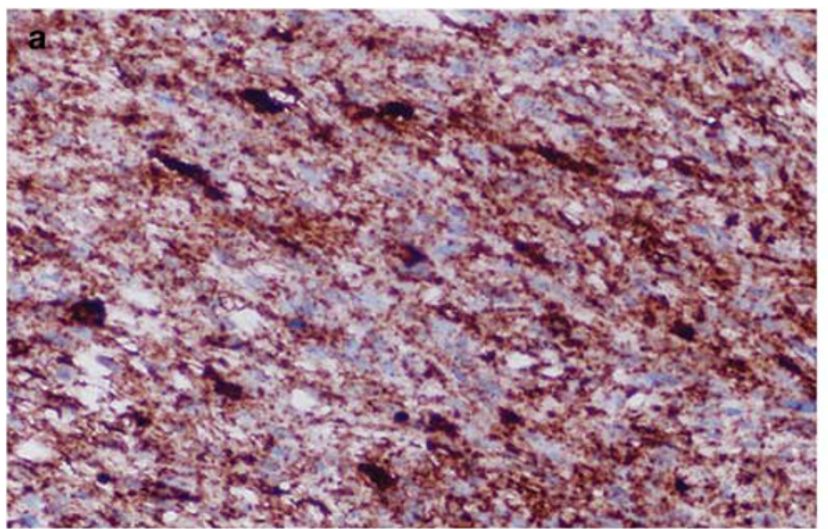

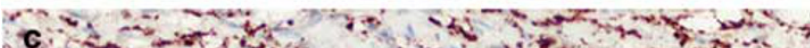

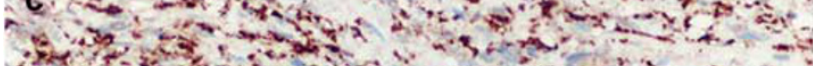

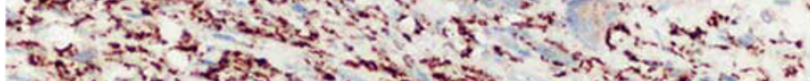

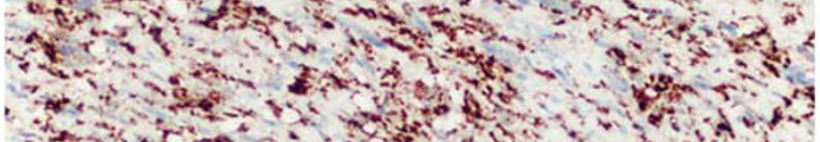

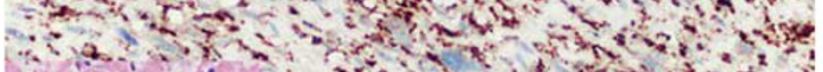

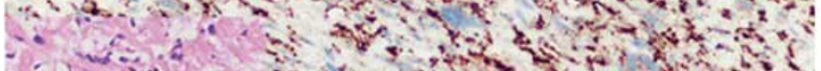

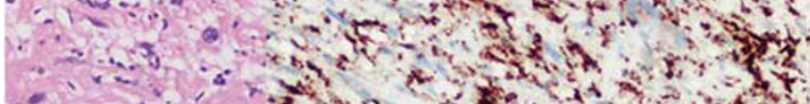

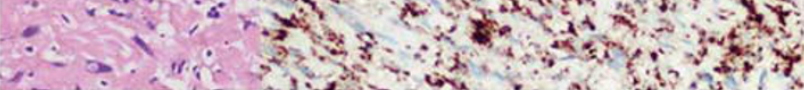
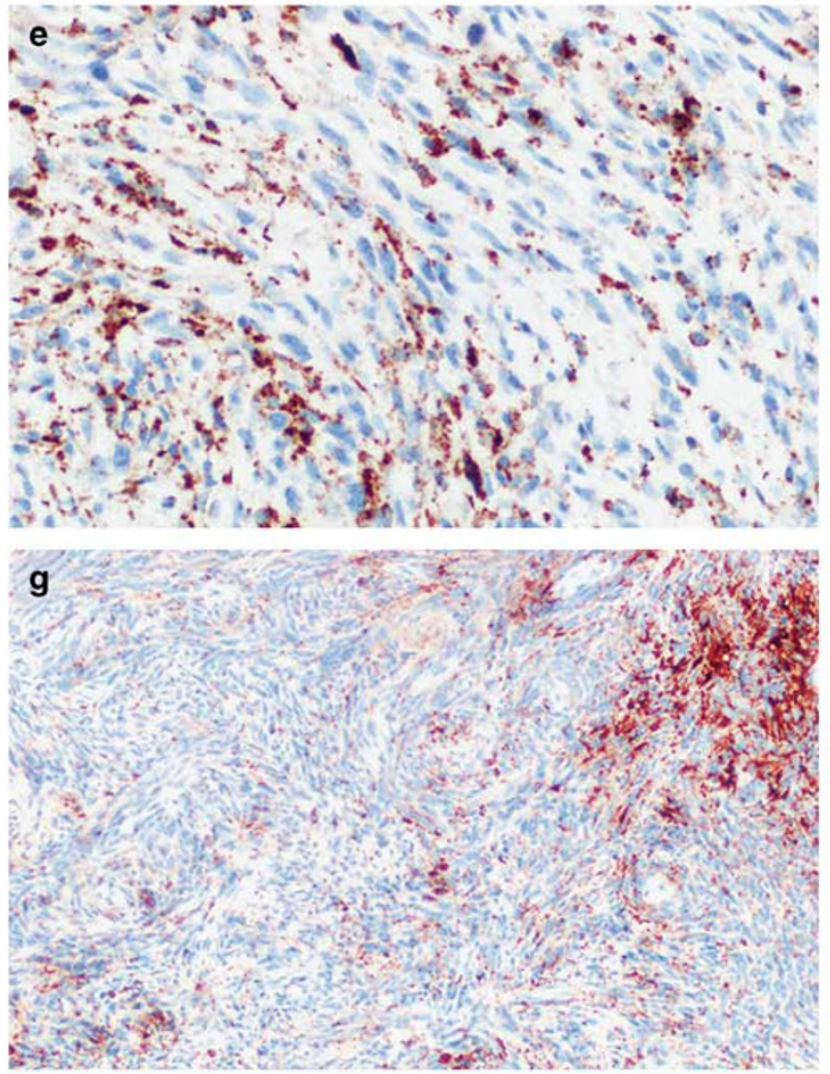

counts of $\geq 10$ mitoses per 10 high-power fields served as a diagnostic feature with high specificity for malignant peripheral nerve sheath tumor. Simi-
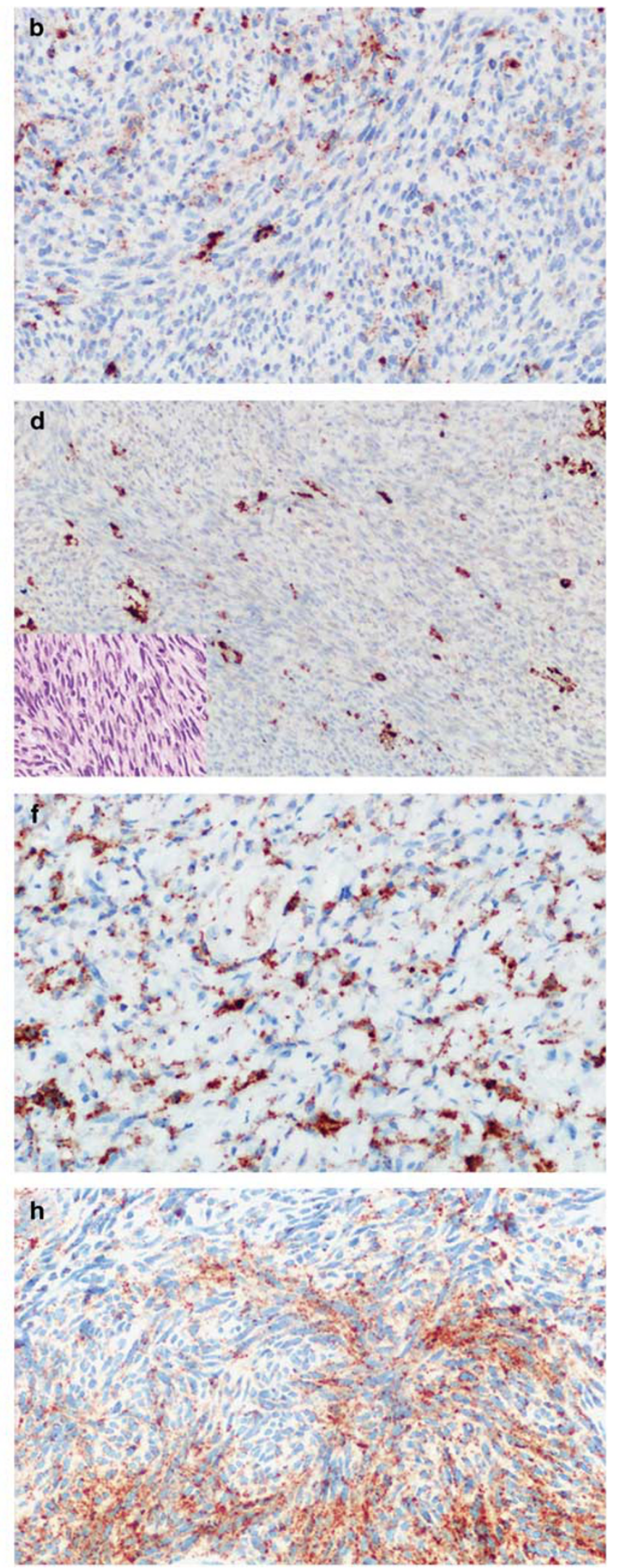
larly, malignant peripheral nerve sheath tumor cases had higher Ki67 proliferation indices, although they overlapped significantly with cellular schwannoma. Data regarding the mitotic activity of cellular schwannomas are variable in the literature, with most cases having $<4$ mitoses per 10 high-power fields. ${ }^{10}$ We had only one patient with a cellular schwannoma containing $\geq 10$ mitoses per 10 highpower fields. This individual had undergone a complete resection with negative margins, and was disease free after 59 months of follow-up.

Immunohistochemical analysis revealed that sensitivities of S100 and SOX10 expression for the diagnosis of malignant peripheral nerve sheath tumor were 41 and $18 \%$, respectively. These results are comparable to previous reports and suggest downregulation of Schwannian markers in malignant peripheral nerve sheath tumor. ${ }^{12,21}$ Although the absence of S100 and SOX10 expression certainly raises the concern for other sarcomas, it is relatively specific for malignant peripheral nerve sheath tumor in the setting of cellular schwannoma versus malignant peripheral nerve sheath tumor. Similarly, diffuse expression with either S100 or SOX10 would strongly suggest cellular schwannoma. Since diffuse S100 expression can also be seen in rare malignant peripheral nerve sheath tumors, this immunostain should only be used in context with other morphological and immunohistochemical features. In agreement with previous reports, survival outcomes were similar for tumors with and without S100 or SOX10 expression. ${ }^{2}$
We found that EGFR expression and/or complete loss of neurofibromin or p16 expression was seen exclusively in malignant peripheral nerve sheath tumor, and combined use of these markers would further increase the sensitivity of malignant peripheral nerve sheath tumor diagnosis. A recent study showed that neurofibromin expression was lost in both neurofibromatosis 1-associated and sporadic malignant peripheral nerve sheath tumors, while it was retained in other spindle cell tumors. ${ }^{16}$ The current study is the first study to partially validate these results, albeit focused specifically on the differential diagnosis with cellular schwannoma. Focal loss of neurofibromin was previously described in three sporadic malignant peripheral nerve sheath tumors ${ }^{16}$ while this pattern was observed in five neurofibromatosis 1-associated malignant peripheral nerve sheath tumors in our series. One of these cases had retained neurofibromin expression in the low-grade malignant peripheral nerve sheath tumor component with neurofibromin loss in the high-grade malignant peripheral nerve sheath tumor component. This pattern may suggest a second event during progression to high grade, which would lead to the loss of protein expression. Loss of neurofibromin expression does not have $100 \%$ correlation with NF1 mutations, raising the possibility of other molecular or epigenetic regulation of its expression. ${ }^{16,22}$ We also found one case of cellular schwannoma with patchy neurofibromin expression. The significance of this expression pattern in an otherwise typical cellular schwan-

Table 4 Sensitivity and specificities of selected stains and combination of stains

\begin{tabular}{|c|c|c|c|c|}
\hline & Sensitivity & Specificity & Area under curve & Confidence interval (95\%) \\
\hline S100 $(-)$ & 58.3 & 100 & 0.757 & $0.639-0.875$ \\
\hline SOX10 $(-)$ & 75 & 100 & 0.878 & $0.790-0.967$ \\
\hline Neurofibromin (-) & 68.4 & 100 & 0.838 & $0.738-0.938$ \\
\hline p16 $(-)$ & 73.2 & 100 & 0.919 & $0.846-0.992$ \\
\hline EGFR $(+)$ & 69 & 100 & 0.878 & $0.790-0.967$ \\
\hline p75NTR $(+)$ & 80 & 69.2 & 0.765 & $0.639-0.892$ \\
\hline EGFR $(+)$ or Neurofibromin $(-)$ & 83.8 & 100 & 0.919 & $0.846-0.992$ \\
\hline EGFR $(+)$ or p16 $(-)$ & 89.2 & 100 & 0.946 & $0.885-1.006$ \\
\hline p16 ( - ) or Neurofibromin $(-)$ & 89.2 & 100 & 0.946 & $0.885-1.006$ \\
\hline EGFR $(+)$ or p16 $(-)$ or Neurofibromin $(-)$ & 91.9 & 100 & 0.959 & $0.907-1.012$ \\
\hline Ki67>20\% & 87.2 & 96.2 & $0.914^{\mathrm{a}}$ & $0.840-0.988$ \\
\hline
\end{tabular}

$(-)$, diffuse loss, expression in $<5 \%$ of tumor cells; $(+)$, positive, expression in $5 \%$ or more of the tumor cells.

${ }^{\mathrm{a}}$ Continues variables (percentage of positive cells) were used to create receiver operating characteristics curve and $20 \%$ was chosen as cutoff.

Figure 5 Patterns of patchy neurofibromin expression. (a, b) High-grade neurofibromatosis I (NF1)-associated malignant peripheral nerve sheath tumor demonstrating focal areas of diffuse neurofibromin expression (a) and other areas of neurofibromin loss (b). (c, d) NF-1 associated malignant peripheral nerve sheath tumor with low-grade component diffusely positive for neurofibromin (c) and high-grade component completely negative for neurofibromin (d). Inset pictures are hematoxylin and eosin stained sections of corresponding components. (e) High-grade NF1-associated malignant peripheral nerve sheath tumor with intermixed positive and negative cells on neurofibromin stain. (f) Low-grade NF1-associated malignant peripheral nerve sheath tumor with intermixed positive and negative cells on neurofibromin stain. ( $\mathbf{g}, \mathbf{h})$ Cellular schwannoma with intermixed positive and negative cells on neurofibromin stain, moderate (g) and high power (h). 
Table 5 Associations of clinical features and the survival results among malignant peripheral nerve sheath cases

\begin{tabular}{|c|c|c|c|c|c|c|}
\hline & \multicolumn{3}{|c|}{$\begin{array}{c}\text { Progression-free } \\
\text { survival }\end{array}$} & \multicolumn{3}{|c|}{$\begin{array}{c}\text { Disease-specific } \\
\text { survival }\end{array}$} \\
\hline & $\begin{array}{c}1 \text {-year } \\
(\%)\end{array}$ & $\begin{array}{c}5 \text {-year } \\
(\%)\end{array}$ & $\mathrm{P}^{\mathrm{a}}$ & $\begin{array}{c}1 \text {-year } \\
(\%)\end{array}$ & $\begin{array}{c}5 \text {-year } \\
(\%)\end{array}$ & $\mathrm{P}^{\mathrm{a}}$ \\
\hline \multicolumn{7}{|l|}{ Gender } \\
\hline Male & 56 & 14.4 & 0.966 & 68.9 & 24 & 0.13 \\
\hline Female & 55.5 & 21.4 & & 81.2 & 37.3 & \\
\hline \multicolumn{7}{|l|}{ Age } \\
\hline$<40$ years & 46.4 & 10 & 0.01 & 78.8 & 31.9 & 0.524 \\
\hline$\geq 40$ years & 74.6 & 34.1 & & 72.3 & 31.5 & \\
\hline \multicolumn{7}{|l|}{ NF1 } \\
\hline Yes & 49.4 & 19.2 & 0.88 & 78.4 & 37.9 & 0.393 \\
\hline No & 57.1 & NA & & 75.9 & 17.3 & \\
\hline \multicolumn{7}{|l|}{ Site } \\
\hline Extremity & 70.7 & 13.9 & 0.917 & 71.7 & 37.8 & 0.6 \\
\hline Non-extremity & 49.3 & 19.5 & & 77.3 & 31.1 & \\
\hline \multicolumn{7}{|c|}{ Extent of resection } \\
\hline Sub-total & 37.4 & 0 & $0.422 t$ & 66.7 & 0 & 0.146 \\
\hline Gross-total & 51.6 & 30.6 & & 80.1 & 42.3 & \\
\hline \multicolumn{7}{|l|}{ Margins } \\
\hline Negative & 59.7 & 39.8 & 0.191 & 66.7 & 38.1 & 0.834 \\
\hline Positive & 33.1 & NA & & 76.5 & 11.5 & \\
\hline \multicolumn{7}{|l|}{ Grade } \\
\hline Low & 85.7 & 51.4 & 0.097 & 100 & 80 & 0.007 \\
\hline High & 51.4 & 11.6 & & 73.4 & 27.2 & \\
\hline \multicolumn{7}{|l|}{ Metastasis } \\
\hline No & 47.2 & 35 & 0.43 & 76.4 & 38.3 & 0.003 \\
\hline Yes & 46.3 & NA & & 65.9 & 5.1 & \\
\hline \multicolumn{7}{|c|}{ Recurrence/Progression } \\
\hline No & & & & 57.8 & 37.5 & 0.883 \\
\hline Yes & & & & 84.8 & 31.3 & \\
\hline
\end{tabular}

Abbreviation: NA, not available, all cases were censored.

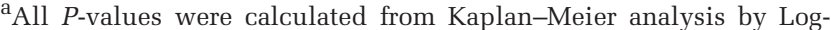
rank method, and $P$-values in bold are significant.

noma is unclear, although it suggests that some interpretive caution is required when loss of neurofibromin expression is not complete.

Loss of p16 expression in malignant peripheral nerve sheath tumor compared with retained expression in neurofibromas was previously reported in the setting of NF1. ${ }^{23}$ These authors also showed the homozygous deletion of $C D K N 2 A / p 16$ gene by PCR. Later, we reported homozygous deletion of $p 16$ by FISH exclusively in malignant peripheral nerve sheath tumor relative to other morphologicallysimilar spindle cell neoplasms. ${ }^{13}$ Other groups reported that decreased $C D K N 2 A$ gene dosage was associated with metastases and poor survival among malignant peripheral nerve sheath tumors. ${ }^{24}$ More recently, Endo et $a l^{25}$ reported decreased $(<50 \%)$ p16 expression by immunohistochemistry in a group of malignant peripheral nerve sheath tumors, which was associated with poor prognosis.
Table 6 Associations of immunohistochemical profile and the survival results among malignant peripheral nerve sheath cases

\begin{tabular}{|c|c|c|c|c|c|c|}
\hline & \multicolumn{3}{|c|}{ Progression-free survival } & \multicolumn{3}{|c|}{ Disease-specific survival } \\
\hline & $\begin{array}{c}\text { 1-year } \\
(\%)\end{array}$ & $\begin{array}{c}5 \text {-year } \\
(\%)\end{array}$ & $\mathrm{P}^{\mathrm{a}}$ & $\begin{array}{c}1 \text {-year } \\
(\%)\end{array}$ & $\begin{array}{c}5 \text {-year } \\
(\%)\end{array}$ & $\mathrm{P}^{\mathrm{a}}$ \\
\hline \multicolumn{7}{|l|}{$S 100$} \\
\hline Positive & 65.2 & 22.1 & 0.318 & 85.1 & 33.3 & 0.463 \\
\hline Negative & 47.2 & 12.9 & & 70.7 & 31.1 & \\
\hline \multicolumn{7}{|l|}{ SOX10 } \\
\hline Positive & 65.6 & 35 & 0.372 & 83.3 & 46.4 & 0.495 \\
\hline Negative & 52.7 & 13.7 & & 74.3 & 28.9 & \\
\hline \multicolumn{7}{|c|}{ Neurofibromin } \\
\hline Positive & 54.5 & 29.1 & 0.43 & 83.3 & 21.9 & 0.019 \\
\hline Negative & 36.5 & NA & & 69.6 & 15 & \\
\hline \multicolumn{7}{|l|}{ p16 } \\
\hline Positive & 67.7 & 36.3 & 0.218 & 82.9 & 44 & 0.185 \\
\hline Negative & 50.9 & 10.7 & & 74 & 30.4 & \\
\hline \multicolumn{7}{|l|}{ EGFR } \\
\hline Positive & 40.1 & 21.9 & 0.228 & 75 & 25.4 & 0.2 \\
\hline Negative & 67.3 & 17.8 & & 77.4 & 35.9 & \\
\hline \multicolumn{7}{|l|}{$p 75 N T R$} \\
\hline Positive & 46.6 & 28.7 & 0.981 & 75.8 & 24.9 & 0.897 \\
\hline Negative & 50 & NA & & 75 & 18.8 & \\
\hline \multicolumn{7}{|l|}{ SOX2 } \\
\hline Positive & 54.2 & 22.3 & 0.507 & 73.8 & 32.3 & 0.421 \\
\hline Negative & 61.4 & NA & & 88.9 & 32.4 & \\
\hline \multicolumn{7}{|l|}{ p53 } \\
\hline Positive & 55.6 & 26.5 & 0.255 & 81.2 & 26.4 & 0.246 \\
\hline Negative & 16.7 & NA & & 60 & NA & \\
\hline \multicolumn{7}{|l|}{ Ki67, \% } \\
\hline$<20$ & 80 & NA & 0.199 & 80 & NA & 0.048 \\
\hline$\geq 20$ & 42.9 & 21.2 & & 74.2 & 9.1 & \\
\hline
\end{tabular}

Abbreviation: NA, not available, all cases were censored.

a All $P$-values were calculated from Kaplan-Meier analysis by Logrank method, and $P$-values in bold are significant.

They have reported 5-year survival rates of 34 and $69 \%$ in tumors with decreased p16 versus tumors with intact p16 expression. In our data set, retained p16 expression did not have a survival benefit even using a $50 \%$ cutoff (data not shown).

EGFR appears to be an important factor in the development of malignant peripheral nerve sheath tumor, and EGFR expression by immunohistochemistry was reported in $29-62 \%$ of malignant peripheral nerve sheath tumors. ${ }^{24,26,27}$ A separate study showed lack of EGFR expression by immunohistochemistry in schwannomas, ${ }^{28}$ suggesting potential use of EGFR immunohistochemistry as a diagnostic test. EGFR overexpression, and thus detection by immunohistochemistry, may be the result of EGFR gene amplification or activating mutations, and previous studies favor the former. ${ }^{13,24,27}$ In some studies, EGFR overexpression correlates with more aggressive disease course, 
advanced stage at presentation, higher tumor mitotic rates, and poor overall outcome.,26,29 In this regard, the mean disease-free survival was 17.4 months for patients overexpressing EGFR compared with 30.1 months for patients lacking overexpression, with corresponding 5-year overall survival rates of 25 and $52 \%$, respectively. In addition, high EGFR expression using a cutoff value of $30 \%$ was reported as associated with worse progression-free survival and overall survival rates. ${ }^{29}$ In our data set, 5-year disease-specific survival was 35.9 and $25.4 \%$ for tumors with and without EGFR expression, respectively, but this did not reach statistical significance. This lack of association with survival remained even when using the $30 \%$ cutoff value (data not shown).

p75NTR is a transmembrane protein that binds nerve growth factor, and has been used as a marker of neural crest origin. ${ }^{30}$ Bonetti et $a l^{15}$ reported lack of p75NTR expression in non-neoplastic tissues and malignant peripheral nerve sheath tumors compared with the increased expression observed in schwannomas. Other groups reported high rates of expression in both schwannomas and malignant peripheral nerve sheath tumors in addition to numerous other tumors, including synovial sarcomas and rhabdomyosarcomas. ${ }^{31}$ In our study, p75NTR expression was more common in malignant peripheral nerve sheath tumors compared with cellular schwannomas. Among the malignant peripheral nerve sheath tumor cases, the presence of p75NTR expression did not show any association with progression-free survival or disease-specific survival. Larger studies using fresh tumor tissues will be required to evaluate whether there is a dosage effect for p75NTR expression.

SOX2 is a transcription factor that is crucial for maintenance of neural stem cell pluripotency. ${ }^{32}$ A prior study reported SOX2 expression in all schwannomas compared with lack of expression in normal peripheral nerve and traumatic neuromas. ${ }^{33}$ We found that SOX2 was expressed in the majority of both cellular schawannomas and malignant peripheral nerve sheath tumors, but did provide diagnostic value. In addition, we failed to demonstrate a prognostic benefit of SOX2 expression among malignant peripheral nerve sheath tumor cases. Additional studies evaluating SOX2 expression in other benign nerve sheath tumors may provide further understanding of its role in malignant peripheral nerve sheath tumor.

We have also evaluated p53 expression in cellular schwannomas and malignant peripheral nerve sheath tumors, as well as its prognostic role in malignant peripheral nerve sheath tumor patient outcome. Previous studies have yielded variable conclusions. Verdijk et $a 3^{34}$ reported p53 mutation in $24 \%$ of their malignant peripheral nerve sheath tumor cases, while immunohistochemical expression of p53 was increased ( $>20 \%$ labeling) in $21 \%$ of their cases. Others reported increased p53 expression in malignant peripheral nerve sheath tumors compared with neurofibromas using a $30 \%$ cutoff. ${ }^{34}$ There was a significant overlap between cellular schawannoma and malignant peripheral nerve sheath tumors in our series, arguing against a diagnostic role for p53. Expression of p53 protein has also been associated with worse outcome in malignant peripheral nerve sheath tumor. ${ }^{2}$ In our data set, malignant peripheral nerve sheath tumor cases with lack of p53 expression showed worse disease-specific survival, although this did not reach statistical significance.

One of the potential concerns with immunohistochemical markers that tend to be expressed heterogeneously is that small needle biopsies will lead to less reliable interpretations. When comparing whole section versus tissue microarray samples, no significant differences for the majority of the stains were found in our study. Although there was a slightly higher rate of positivity with S100 and SOX10 among whole section, the opposite trend was found for $\mathrm{p} 16$ and SOX2 stains and these differences did not reach statistical significance. In contrast, EGFR expression was found significantly less often in tissue microarrays, suggesting that the sensitivity of EGFR staining may be low in needle biopsies or other small specimens. In addition, there were occasional cases with core to core variability that could potentially lead to misclassification on a single core sample. As such, greater care must be exercised when interpreting such immunostains in particularly limited samples.

In summary, the presence of Schwannian whorls, a peritumoral capsule, subcapsular lymphocytes, macrophage-rich infiltrates, and the absence of fascicles favor the diagnosis of cellular schwannoma, while the presence of perivascular hypercellularity, tumor herniation into vascular lumens, and necrosis favor malignant peripheral nerve sheath tumor. Complete loss of SOX10, neurofibromin or p16 expression, or the presence of EGFR immunoreactivity strongly favor malignant peripheral nerve sheath tumor. Taken together, immunohistochemistry is useful in the differential diagnosis of malignant peripheral nerve sheath tumor and cellular schwannoma. In addition to the most commonly utilized markers of S100 and SOX10, immunohistochemistry for EGFR, p16, and neurofibromin shows the highest sensitivities and specificities in this diagnostic setting.

\section{Acknowledgments}

This project was supported by Residents' Teaching and Research Endowments from University of California San Francisco, Department of Pathology. This study was presented in part at the United States and Canadian Academy of Pathology 103rd Annual Meeting in San Diego, CA, 4 March 2014 (Abstract 1809).

\section{Disclosure/conflict of interest}

The authors declare no conflict of interest. 


\section{References}

1 Louis DN, Ohgaki H, Wiestler OD, Cavenee WK (eds). WHO Classification of Tumours of Central Nervous System, 4th edn. IARC Press: Lyon, 2007.

2 Zou C, Smith KD, Liu J, et al. Clinical, pathological, and molecular variables predictive of malignant peripheral nerve sheath tumor outcome. Ann Surg 2009; 249:1014-1022.

3 Fan Q, Yang J, Wang G. Clinical and molecular prognostic predictors of malignant peripheral nerve sheath tumor. Clin Transl Oncol 2014;16:191-199.

4 Kolberg M, Holand M, Agesen TH, et al. Survival metaanalyses for $>1800$ malignant peripheral nerve sheath tumor patients with and without neurofibromatosis type 1. Neuro Oncol 2013;15:135-147.

5 Woodruff JM, Godwin TA, Erlandson RA, et al. Cellular schwannoma: a variety of schwannoma sometimes mistaken for a malignant tumor. Am J Surg Pathol 1981;5:733-744.

6 Fletcher CD, Davies SE, McKee PH. Cellular schwannoma: a distinct pseudosarcomatous entity. Histopathology 1987;11:21-35.

7 Seppala MT, Haltia MJ. Spinal malignant nerve-sheath tumor or cellular schwannoma? A striking difference in prognosis. J Neurosurg 1993;79:528-532.

8 Casadei GP, Scheithauer BW, Hirose T, et al. Cellular schwannoma. A clinicopathologic, DNA flow cytometric, and proliferation marker study of 70 patients. Cancer 1995;75:1109-1119.

9 Henke AC, Salomao DR, Hughes JH. Cellular schwannoma mimics a sarcoma: an example of a potential pitfall in aspiration cytodiagnosis. Diagn Cytopathol 1999;20:312-316.

10 White W, Shiu MH, Rosenblum MK, et al. Cellular schwannoma. A clinicopathologic study of 57 patients and 58 tumors. Cancer 1990;66:1266-1275.

11 Perry A, Brat D (eds). Practical Surgical Neuropathology: A Diagnostic Approach, 1st edn. Churchill Livingstone/Elsevier: Philadelphia, PA, 2010;620p.

12 Karamchandani JR, Nielsen TO, van de Rijn M, et al. Sox10 and $\mathrm{S} 100$ in the diagnosis of soft-tissue neoplasms. Appl Immunohistochem Mol Morphol 2012;20:445-450.

13 Perry A, Kunz SN, Fuller CE, et al. Differential NF1, p16, and EGFR patterns by interphase cytogenetics (FISH) in malignant peripheral nerve sheath tumor (MPNST) and morphologically similar spindle cell neoplasms. J Neuropathol Exp Neurol 2002;61:702-709.

14 Kang Y, Pekmezci M, Folpe AL, et al. Diagnostic utility of SOX10 to distinguish malignant peripheral nerve sheath tumor from synovial sarcoma, including intraneural synovial sarcoma. Mod Pathol 2014;27: 55-61.

15 Bonetti B, Panzeri L, Carner M, et al. Human neoplastic Schwann cells: changes in the expression of neurotrophins and their low-affinity receptor p75. Neuropathol Appl Neurobiol 1997;23:380-386.

16 Reuss DE, Habel A, Hagenlocher C, et al. Neurofibromin specific antibody differentiates malignant peripheral nerve sheath tumors (MPNST) from other spindle cell neoplasms. Acta Neuropathol 2014;127: 565-572.

$17 \mathrm{Yu} \mathrm{J}$, Deshmukh H, Payton JE, et al. Array-based comparative genomic hybridization identifies CDK4 and FOXM1 alterations as independent predictors of survival in malignant peripheral nerve sheath tumor. Clin Cancer Res 2011;17:1924-1934.

18 Ducatman BS, Scheithauer BW, Piepgras DG, et al. Malignant peripheral nerve sheath tumors. A clinicopathologic study of 120 cases. Cancer 1986;57:2006-2021.

19 Gutmann DH, Aylsworth A, Carey JC, et al. The diagnostic evaluation and multidisciplinary management of neurofibromatosis 1 and neurofibromatosis 2 . JAMA 1997;278:51-57.

20 Alberghini M, Zanella L, Bacchini P, et al. Cellular schwannoma: a benign neoplasm sometimes overdiagnosed as sarcoma. Skeletal Radiol 2001;30:350-353.

21 Miller SJ, Rangwala F, Williams J, et al. Large-scale molecular comparison of human schwann cells to malignant peripheral nerve sheath tumor cell lines and tissues. Cancer Res 2006;66:2584-2591.

22 Gutmann DH, McLellan MD, Hussain I, et al. Somatic neurofibromatosis type 1 (NF1) inactivation characterizes NF1-associated pilocytic astrocytoma. Genome Res 2013;23:431-439.

23 Nielsen GP, Stemmer-Rachamimov AO, Ino Y, et al. Malignant transformation of neurofibromas in neurofibromatosis 1 is associated with CDKN2A/p16 inactivation. Am J Pathol 1999;155:1879-1884.

24 Holtkamp N, Malzer E, Zietsch J, et al. EGFR and erbB2 in malignant peripheral nerve sheath tumors and implications for targeted therapy. Neuro Oncol 2008;10:946-957.

25 Endo M, Kobayashi C, Setsu N, et al. Prognostic significance of p14ARF, p15INK4b, and p16INK4a inactivation in malignant peripheral nerve sheath tumors. Clin Cancer Res 2011;17:3771-3782.

26 Keizman D, Issakov J, Meller I, et al. Expression and significance of EGFR in malignant peripheral nerve sheath tumor. J Neurooncol 2009;94:383-388.

27 Tawbi H, Thomas D, Lucas DR, et al. Epidermal growth factor receptor expression and mutational analysis in synovial sarcomas and malignant peripheral nerve sheath tumors. Oncologist 2008;13:459-466.

28 Prayson RA, Yoder BJ, Barnett GH. Epidermal growth factor receptor is not amplified in schwannomas. Ann Diagn Pathol 2007;11:326-329.

$29 \mathrm{Du}$ X, Yang J, Ylipaa A, et al. Genomic amplification and high expression of EGFR are key targetable oncogenic events in malignant peripheral nerve sheath tumor. J Hematol Oncol 2013;6:93.

30 Lazova R, Tantcheva-Poor I, Sigal AC. P75 nerve growth factor receptor staining is superior to S100 in identifying spindle cell and desmoplastic melanoma. J Am Acad Dermatol 2010;63:852-858.

31 Fanburg-Smith JC, Miettinen M. Low-affinity nerve growth factor receptor (p75) in dermatofibrosarcoma protuberans and other nonneural tumors: a study of 1,150 tumors and fetal and adult normal tissues. Hum Pathol 2001;32:976-983.

32 Widera D, Heimann P, Zander C, et al. Schwann cells can be reprogrammed to multipotency by culture. Stem Cells Dev 2011;20:2053-2064.

33 Shivane A, Parkinson DB, Ammoun S, et al. Expression of c-Jun and Sox-2 in human schwannomas and traumatic neuromas. Histopathology 2013;62:651-656.

34 Verdijk RM, den Bakker MA, Dubbink HJ, et al. TP53 mutation analysis of malignant peripheral nerve sheath tumors. J Neuropathol Exp Neurol 2010;69:16-26. 